\title{
Body-Centered-Cubic Ni and Its Magnetic Properties
}

\author{
C. S. Tian, D. Qian, D. Wu, R. H. He, Y.Z. Wu, W. X. Tang, L. F. Yin, Y. S. Shi, G. S. Dong, and X. F. Jin* \\ Surface Physics Laboratory, Fudan University, Shanghai 200433, China \\ X. M. Jiang, ${ }^{1}$ F. Q. Liu, ${ }^{1}$ H. J. Qian, ${ }^{1}$ K. Sun, ${ }^{2}$ L. M. Wang, ${ }^{2}$ G. Rossi, ${ }^{3}$ Z. Q. Qiu, ${ }^{4}$ and J. Shi ${ }^{5}$ \\ ${ }^{1}$ National Synchrotron Radiation Laboratory, Beijing 310027, China \\ ${ }^{2}$ Nuclear Engineering and Radiological Sciences, University of Michigan, Ann Arbor Michigan 48109, USA \\ ${ }^{3}$ Laboratorio Nazionale TASC-INFM, Strada Statale 14, km 163.5 Basovizza, I-34012 Trieste, Italy \\ ${ }^{4}$ Department of Physics, University of California, Berkeley, California 94720, USA \\ ${ }^{5}$ Department of Physics, University of Utah, Salt Lake City, Utah 84112, USA
}

(Received 18 November 2004; published 7 April 2005)

\begin{abstract}
The body-centered-cubic (bcc) phase of $\mathrm{Ni}$, which does not exist in nature, has been achieved as a thin film on $\operatorname{GaAs}(001)$ at $170 \mathrm{~K}$ via molecular beam epitaxy. The bcc $\mathrm{Ni}$ is ferromagnetic with a Curie temperature of $456 \mathrm{~K}$ and possesses a magnetic moment of $0.52 \pm 0.08 \mu_{B} /$ atom. The cubic magnetocrystalline anisotropy of bcc $\mathrm{Ni}$ is determined to be $+4.0 \times 10^{5} \mathrm{ergs} \cdot \mathrm{cm}^{-3}$, as opposed to $-5.7 \times$ $10^{4} \mathrm{ergs} \cdot \mathrm{cm}^{-3}$ for the naturally occurring face-centered-cubic (fcc) Ni. This sharp contrast in the magnetic anisotropy is attributed to the different electronic band structures between bcc $\mathrm{Ni}$ and fcc $\mathrm{Ni}$, which are determined using angle-resolved photoemission with synchrotron radiation.
\end{abstract}

DOI: 10.1103/PhysRevLett.94.137210

PACS numbers: 75.30. $-\mathrm{m}, 81.20 .-\mathrm{n}$

$\mathrm{Fe}, \mathrm{Co}$, and Ni rank as the top three important ferromagnetic elements in the periodic table that have accumulated extensive literature. These three elements assume bodycentered-cubic (bcc), hexagonal-close-packed (hcp), and face-centered-cubic (fcc) structures, respectively, in their thermodynamically stable phases at room temperature. To develop a deep understanding of the magnetism origin, a great effort has been made in obtaining different crystallographic structures of $\mathrm{Fe}, \mathrm{Co}$, and $\mathrm{Ni}$. Using molecular beam epitaxy, the fcc phase of both Fe and Co [1], which exists only at high temperatures in bulk form, can be stabilized on appropriately chosen nonmagnetic substrates at room temperature. Even the non-naturally occurring bcc Co has been achieved in the laboratory [2]. These advances in materials synthesis have opened up a unique opportunity for the investigation of some long-standing problems in itinerant magnetism [3]. For example, bcc Fe is a well-known prototype ferromagnet; however, fcc Fe can exhibit an antiferromagnetic (spin-density-wave) [4] phase. These discoveries on the magnetic properties that are associated with metastable structural phases have greatly advanced our knowledge on the origin of magnetism. In spite of the fact that both $\mathrm{Fe}$ and $\mathrm{Co}$ can have either bcc or fcc structure, it is puzzling why Ni seems to dwell rigidly only in the fcc structure. It has remained an experimental challenge to synthesize and investigate the bcc phase of Ni.

Theoretically, it is predicted by Moruzzi et al. that bcc $\mathrm{Ni}$ at equilibrium (with a lattice constant of $0.2773 \mathrm{~nm}$ ) would be paramagnetic, but a transition to a ferromagnetic state would occur upon expansion of the lattice beyond $0.2815 \mathrm{~nm}$ [5]. However, it is found by Guo et al. that the paramagnetic-ferromagnetic transition happens at $0.2730 \mathrm{~nm}$, and bcc Ni at $0.280 \mathrm{~nm}$ is already ferromagnetic with a magnetic moment of $0.53 \mu_{B} /$ atom [6].

A crucial step forward in experiment is the successful preparation of bcc $\mathrm{Ni}$ on $\mathrm{Fe}(001)$ by Heinrich et al. [7], and is also confirmed independently by Wang et al. [8]. They both agree that the bcc Ni structure can exist only up to 6 atomic layers (less than 3 unit cells) and the structure of thicker Ni films appears to be more complicated, as called the "modified bcc Ni phase" [9]. In addition, it is found by Bland and Heinrich et al. that 3 monolayers (ML) of bcc Ni on $\mathrm{Fe}(001)$ is ferromagnetic with a magnetic moment of $(0.55-0.80) \mu_{B} /$ atom $[10,11]$, while a value of $(0.4 \pm$ 0.45) $\mu_{B}$ /atom is obtained indirectly from Brookes et al. [12]. But the Curie temperatures of the ultrathin bcc Ni films ( $<6 \mathrm{ML})$ are contradictory between the two sets of experiments: Heinrich et al. found it must be lower than $77 \mathrm{~K}$ [9] while Brookes declared that it is higher than $300 \mathrm{~K}$ [12]. Furthermore, an anomalously strong fourfold inplane magnetic anisotropy is realized for the modified bcc Ni phase on $\mathrm{Fe}(001)$, which is attributed to a nonmagnetocrystalline origin [9].

However, it is shown theoretically [13] as well as experimentally $[9,10,14]$ that there exist strong hybridization or magnetic coupling effects at the $\mathrm{Ni} / \mathrm{Fe}$ interface. This would lead to the fact that all the magnetic properties obtained in the previous experiments are not the intrinsic properties of bcc Ni itself; the contributions from Fe cannot be excluded. For example, the Curie temperature of $\mathrm{Ni}$ ultrathin films might be enhanced significantly by the interface coupling [15], the ferromagnetic ordering of bcc Ni might be induced by $\mathrm{Fe}$ as similar to the $\mathrm{Fe} / \mathrm{Pd}$ case [16], and the magnetic moment of bcc Ni might be greatly enhanced from its intrinsic value because of the 
strong coupling with $\mathrm{Fe}[10,13]$. Therefore the intrinsic magnetic nature of bcc $\mathrm{Ni}$ is still an open question unless a magnetically isolated bcc Ni phase is prepared and characterized. An earlier experiment indicated that $\mathrm{Ni}$ film grown on GaAs(100) may have a bcc structure with some interesting magnetic behaviors [17]. In this Letter, we investigate the $\mathrm{Ni} / \mathrm{GaAs}(100)$ system with a multitechnique approach, including high resolution transmission electron microscope (HRTEM), grazing angle x-ray diffraction (XRD), superconducting quantum interference device (SQUID), angle-resolved photoemission electron spectroscopy (ARPES), and magneto-optic Kerr effect (MOKE) measurements. Here are the main results. (i) It is identified unambiguously that a magnetically isolated Ni thin film $(d<3.5 \mathrm{~nm})$ epitaxially grown on $\mathrm{GaAs}(001)$ at $170 \mathrm{~K}$ has a bcc structure with a lattice constant of $a=0.282 \mathrm{~nm}$. (ii) The bcc $\mathrm{Ni}$ phase has a magnetic moment of $(0.52 \pm 0.08) \mu_{B}$ /atom with the Curie temperature of $456 \mathrm{~K}$. (iii) The cubic magnetocrystalline anisotropy of the bcc $\mathrm{Ni}$ is determined to be $K_{1}=$ $+4.0 \times 10^{5} \mathrm{ergs} \cdot \mathrm{cm}^{-3}$. (iv) Different electronic energy dispersions of bcc and fcc Ni along the $\langle 100\rangle$ direction are clearly observed in the experiment, providing a basis for further theoretical investigations to explain why the bcc and fcc Ni phases have the opposite behaviors in cubic magnetocrystalline anisotropy.

Clean and ordered $\mathrm{GaAs}(001)$ substrates are prepared by argon bombardment and annealing at $830 \mathrm{~K}$, which show a sharp $(4 \times 6)$ LEED pattern and have large atomically flat terraces, visible under a scanning tunneling microscope (STM). A substrate temperature of $170 \mathrm{~K}$ is found to be the optimal growth condition to achieve the thickest bcc Ni films as well as an atomically sharp interface between $\mathrm{Ni}$ and $\operatorname{GaAs}(001)$; the pure single-crystalline bcc Ni film can be stabilized up to $3.5 \mathrm{~nm}$ thick. As the film becomes thicker, the Ni overlayer on top of the pure bcc Ni develops into a more complicated structure, as some extra and complicated diffraction features start to appear. The samples for ex situ measurements are capped with $2 \mathrm{~nm}$
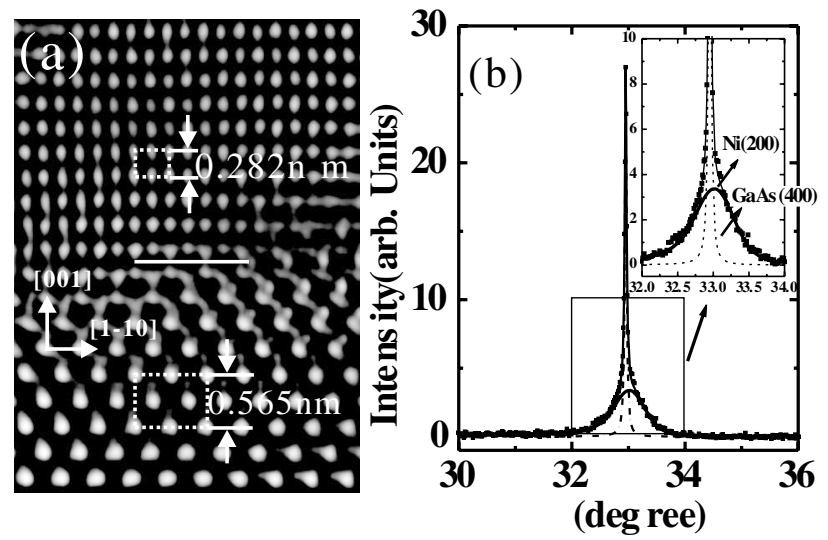

FIG. 1. (a) Fourier-filtered HRTEM image of $3.5 \mathrm{~nm}$ bcc $\mathrm{Ni} / \mathrm{GaAs}(001)$ with the electron beam along the [110] direction. (b) Grazing angle XRD spectrum with incident angle at $0.2^{\circ}$.
Au before being taken out of the UHV. The grazing angle XRD experiment was performed at the National Synchrotron Radiation Laboratory in Beijing. Angleresolved photoemission measurements were performed both in Beijing and at the APE-INFM beam line of ELETTRA in Trieste.

Figure 1(a) shows a cross-sectional HRTEM image of $\mathrm{Ni}(3.5 \mathrm{~nm}) / \mathrm{GaAs}(001)$ with the electron beam along the [110] direction of GaAs. The image was recorded digitally by a JEOL 2010F STEM/TEM analytical electron microscope and analyzed using a Digital Micrograph software. The upper and lower parts in the figure represent bcc-Ni and GaAs substrates, respectively, with the white line showing the interfacial boundary. The unit cells in both regions are also drawn, from which the $\mathrm{Ni}$ is unambiguously identified to have a bcc structure [18] with a lattice constant of $0.28 \mathrm{~nm}$. The relative orientation between the $\mathrm{Ni}$ and $\mathrm{GaAs}$ crystals is bcc $\mathrm{Ni}[001] \| \mathrm{GaAs}[001]$, bcc Ni[100] \| GaAs[100], and bcc Ni[110] \| GaAs[110]. This result is further confirmed by a synchrotron radiationbased grazing angle x-ray diffraction experiment. Figure 1(b) shows an x-ray diffraction spectrum taken at the photon energy of $8.052 \mathrm{keV}$ with an incident angle of $0.2^{\circ}$. In addition to the expected dominant $\operatorname{GaAs}(400)$ diffraction peak, there is a clear broad peak superimposed on top of the narrow GaAs(400) peak [see the inset of Fig. 1(b)]. This broad peak is a fingerprint of the bec $\mathrm{Ni}(200)$ diffraction from which an in-plane lattice constant of $a=0.282 \mathrm{~nm}$ is readily derived for bcc Ni. The absence of the fcc Ni diffraction peaks at $\theta \sim 25.9^{\circ}$ for (200) and at $\theta \sim 38.1^{\circ}$ for (220) (not shown in the figure) proves that the Ni film has only the single-crystal bcc phase.

The magnetism of bcc $\mathrm{Ni}$ and its Curie temperature are studied in situ by means of MOKE measurement, by using a semiconductor laser with the wavelength of $670 \mathrm{~nm}$ and a beam size of $0.3 \mathrm{~mm}$. Figure 2(a) shows the MOKE remanence at $120 \mathrm{~K}$ as a function of film thickness measured

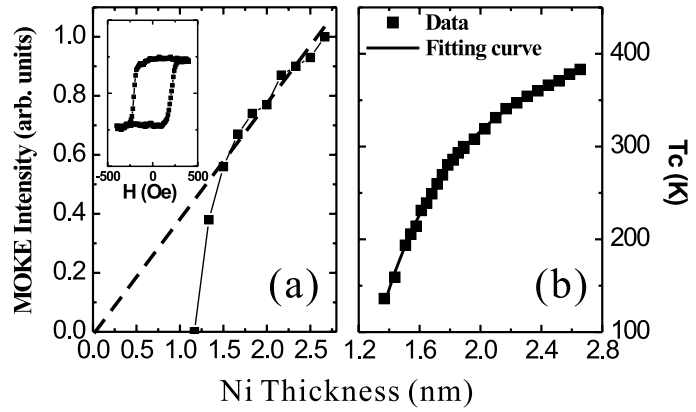

FIG. 2. (a) MOKE intensity at remanence as a function of bcc Ni thickness for a wedged sample measured at $120 \mathrm{~K}$, together with a representative hysteresis loop in the inset. (b) The filled squares represent the experimentally determined Curie temperature as a function of film thickness, and the solid line is a theoretical fit to Eq. (1) with $T_{c}(\infty)=456 \mathrm{~K}, \lambda=2.23$, and $c=1.17 \mathrm{~nm}$. 
from a $\mathrm{Ni}$ wedge with the thickness change from 0.8 to $3.0 \mathrm{~nm}$ over a $1.0 \mathrm{~cm}$ long sample, together with a representative MOKE hysteresis loop in the inset. Similar to Fe on $\operatorname{GaAs}(001)$ [19], no interfacial magnetic dead layers are found in bcc $\mathrm{Ni} / \mathrm{GaAs}(001)$ grown at $170 \mathrm{~K}$, as seen from the extrapolated dashed line Fig. 2(a) which passes through the origin. The vanishing MOKE signal of the bcc Ni below a critical thickness of $d_{c} \sim 1.2 \mathrm{~nm}$, i.e., the paramagnetic-to-ferromagnetic transition at $T=120 \mathrm{~K}$, is caused by the well-known finite size scaling effect of the Curie temperature in ultrathin films. Compared with the value of $d_{c} \sim 0.6 \mathrm{~nm}$ at $120 \mathrm{~K}$ for fcc $\mathrm{Ni}$ on $\mathrm{Cu}(001)$ [20], the greater $d_{c}$ value here implies that the Curie temperature of the bcc Ni film is lower than that of the fcc Ni film at the same thickness. In order to extrapolate the Curie temperature for bulk bcc Ni, we measured the $T_{c}$ versus $d_{c}$ curve [Fig. 2(b)] from the bcc Ni wedged sample by gradually changing the sample temperatures from 120 to $380 \mathrm{~K}$ (below which the reversible results are obtained). From the well-known finite size scaling law for magnetic phase transition [21],

$$
\left[T_{c}(\infty)-T_{c}(d)\right] / T_{c}(\infty)=(c / d)^{\lambda}
$$

where $T_{c}(\infty)$ is the Curie temperature in the bulk limit, $d$ is the film thickness, and $\lambda$ is a critical exponent whose value reflects the universality class of the phase transition, we fitted the experimental data [solid line in Fig. 2(b)] using Eq. (1) and obtained a Curie temperature of $456 \mathrm{~K}$ for bulk bcc $\mathrm{Ni}$, which is indeed significantly lower than $627 \mathrm{~K}$, the Curie temperature of bulk fcc Ni. The magnetization of bcc $\mathrm{Ni}$ (capped with $2 \mathrm{~nm} \mathrm{Au}$ ) was further measured ex situ at $5 \mathrm{~K}$ by SQUID, which yields a value of $(0.52 \pm$ 0.08) $\mu_{B} /$ atom, in good agreement with the theoretical predicted value of $0.54 \mu_{B}$ /atom at the lattice constant of $a=0.282 \mathrm{~nm}[6]$.

The success in producing single-crystalline bcc $\mathrm{Ni}$ on $\mathrm{GaAs}(001)$ makes it possible to quantitatively determine the bcc Ni cubic magnetocrystalline anisotropy $K_{1}$. We use the so-called ROTMOKE (MOKE with a rotating magnetic

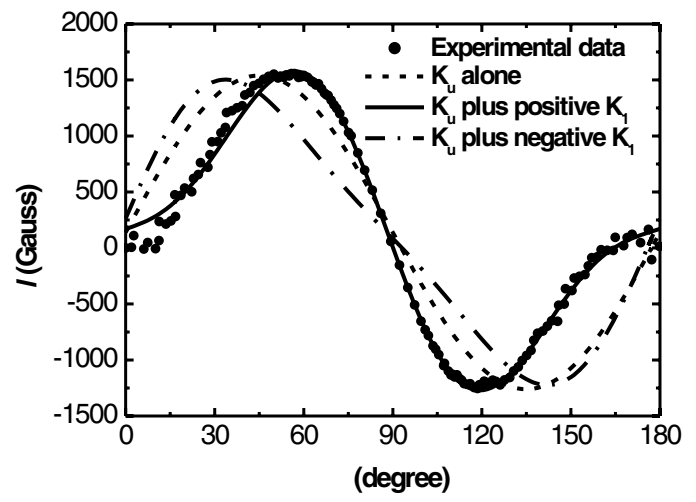

FIG. 3. MOKE-based torque as a function of the magnetization directions, together with different theoretical fits, as described in the inset as well as in the text. field) technique [22] to address this problem. Similar to the procedure of determining the anisotropies of epitaxially grown $\mathrm{Fe}_{x} \mathrm{Cu}_{1-x}$ films on $\mathrm{GaAs}(001)$ [23], we obtain the MOKE-based torque as a function of the in-plane magnetic field $(H=5000 \mathrm{Oe})$ for $3.5 \mathrm{~nm}$ bcc-Ni/GaAs$(001)$ (black dots in Fig. 3). At a glance the $180^{\circ}$ periodicity in the oscillation suggests a typical uniaxial magnetic anisotropy, which is always found in magnetic film on a III-V semiconductor and the origin is not clear up to now [24]. However, a fit with the uniaxial anisotropy $K_{u}$ alone does not agree with the experimental curve (the dashed line in Fig. 3), directly indicating that the contribution of the magnetic cubic anisotropy $K_{1}$ is also important. Indeed the fit is significantly improved when both $K_{u}$ and $K_{1}$ terms are included. The excellent fit (the solid line in Fig. 3) yields the uniaxial and fourfold magnetic anisotropic fields of $H_{k u}=2300 \mathrm{Oe}$ and $H_{k 1}=1800 \mathrm{Oe}$, respectively, $\left(H_{k i}=2 K_{i} / M_{s}\right)$. Using the foregoing obtained magnetic moment of bec Ni, the magnetic cubic anisotropy $K_{1}$ is determined to be $+4.0 \times 10^{5} \mathrm{ergs} \cdot \mathrm{cm}^{-3}$. It is important to note that the positive sign of $K_{1}$ indicates that bcc Ni has the same cubic magnetic easy axis as bcc Fe, but opposite to that of fcc Ni [25]. To verify the sign of the cubic anisotropy, we intentionally fix the sign of $H_{k 1}$ to be negative in the fitting, but the result turns out to be much worse as seen by the dash-dotted line in Fig. 3. Therefore we conclude that bcc Ni has a positive cubic magnetocrystalline anisotropy $K_{1}$, and its magnitude is much greater than that of naturally occurring fcc $\mathrm{Ni}\left(K_{1}=\right.$ $\left.-5.7 \times 10^{4} \mathrm{ergs} \cdot \mathrm{cm}^{-3}\right)$ but comparable with that of bcc $\mathrm{Fe}\left(K_{1}=+4.7 \times 10^{5} \mathrm{ergs} \cdot \mathrm{cm}^{-3}\right)$. In addition, it should be mentioned that the presence of the uniaxial anisotropy in thick bcc Ni films could suggest that the bcc lattice has some degree of in-plane shear strain, similar to that of thick bcc Fe layers on GaAs(001) [26].

The sharp contrast in the magnetic anisotropy between fcc $\mathrm{Ni}$ and bcc Ni should also be reflected in their electronic band structures, although the correlation is not trivial at all. It has long been a problem for ab initio calculations to account correctly for the electronic band structure of fcc Ni [27]; we believe that it is interesting to provide here the first experimental band structure result for bcc Ni. Accordingly, an angle-resolved photoemission spectroscopy study by variable photon energy $(h \nu)$ was performed at $120 \mathrm{~K}$ on $1.6-\mathrm{nm}$ thick bec $\mathrm{Ni} / \mathrm{GaAs}(001)$ and fcc $\mathrm{Ni} / \mathrm{Cu}(001)$ films, respectively, along the $\langle 001\rangle$ direction (normal emission). As shown in Fig. 4, two main features can be identified as the difference in the valence band structures between the fcc and bcc Ni phases. First, a contrast in the line shape is found for each pair of energy distribution curves (EDC's) obtained at the same $h \nu$ on the two crystals, as shown in the inset for a couple of representative curves measured at $h \nu=45 \mathrm{eV}$. A multicomponent nature is more readily identified in the fcc Ni spectra (left panel), whereas a seemingly single broad peak persists in the whole $h \nu$ range in the bcc case (right panel). Second, in contrast to the apparent band dispersion for fcc $\mathrm{Ni}$, the 


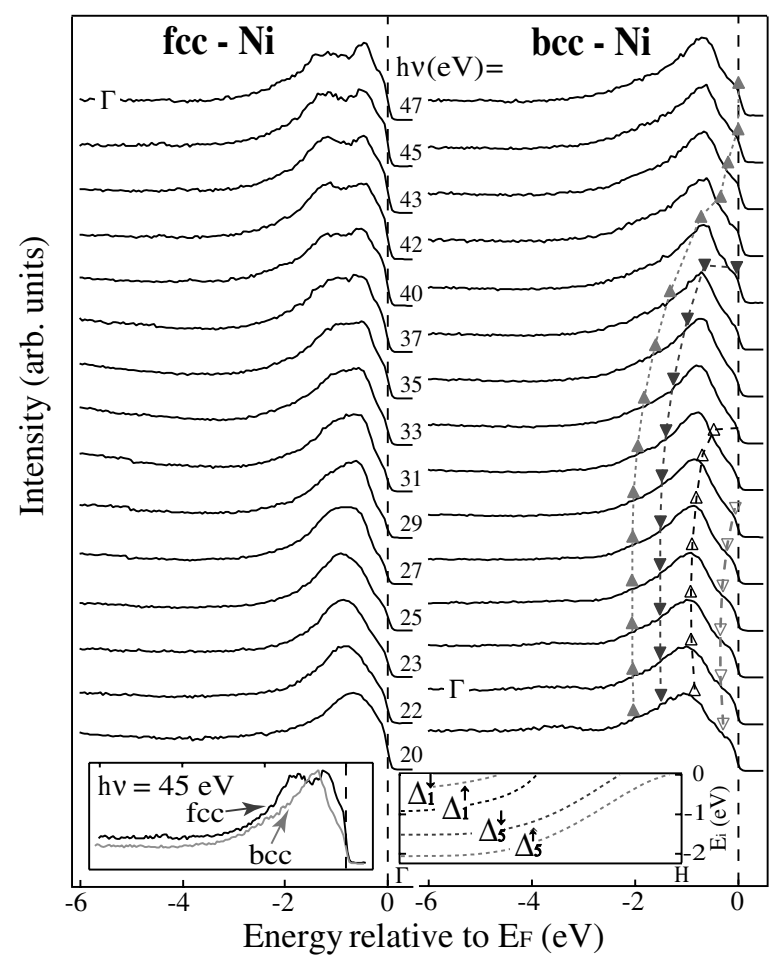

FIG. 4. Normal emission spectra of (left) fcc Ni/Cu(001) and (right) bcc Ni/GaAs(001). Insets: (left) a couple of representative EDC curves for fcc and bcc Ni measured at $h \nu=45 \mathrm{eV}$; (right) calculated spin-resolved initial states of bcc Ni which are involved in the direct transitions. The dashed lines serve as guides to the eye.

valence bands of bcc Ni exhibit a virtually nondispersive character other than a discernible Fermi crossing of some low-lying states. In order to gain further insight into the band structure of bcc Ni, we have calculated the $E(k)$ banddispersion curves using the first- principles linearized augmented plane-wave method with the local spin density approximation. The four low-lying bands, which contribute to the photoemission data here under the dipole transition selection rule, are shown in the inset and are also displayed by the triangles over the experimental curves. A good agreement between the theory and the experiment is obtained on the main EDC features, their dispersions, as well as a persistent Fermi cutoff. In addition, the other features in the experimental curves of bcc Ni can also be explained after taking into account the transitions beyond the first in-plane Brillouin zone, which are not shown here but will be described elsewhere in detail [28].

In summary, we have achieved for the first time magnetically isolated single-crystalline bcc $\mathrm{Ni}$ film on $\mathrm{GaAs}(001)$. We have experimentally determined its ferromagnetic magnetic moment $\left[(0.52 \pm 0.08) \mu_{B} /\right.$ atom $]$ and its Curie temperature $(456 \mathrm{~K})$. bcc Ni exhibits a distinct positive cubic anisotropy of $K_{1}$ and different electron structures to that of fcc $\mathrm{Ni}$.
This work was supported by the National Natural Science Foundation of China, by the 973 Project of the Science and Technology Ministry of China, and by the Shanghai Science and Technology Committee. C.S.T. acknowledges the help of L. Giovannelli, I. Vobornik, and G. Panaccione at INFM, and partial support from the Italian Ministry of Foreign Affairs.

*Corresponding author.

Electronic address: xfjin@fudan.edu.cn

[1] See, e.g., D. Pescia et al., Phys. Rev. Lett. 58, 2126 (1987); 58, 933 (1987).

[2] G. A. Prinz, Phys. Rev. Lett. 54, 1051 (1985); E. Gu et al., Phys. Rev. B 52, 14704 (1995); Y.Z. Wu et al., Phys. Rev. B 57, 11935 (1998).

[3] L. M. Falicov et al., J. Mater. Res. 5, 1299 (1990).

[4] Y. Tsunoda, J. Phys. Condens. Matter 1, 10427 (1989); D. Qian et al., Phys. Rev. Lett. 87, 227204 (2001).

[5] V.L. Moruzzi et al., Phys. Rev. Lett. 57, 2211 (1986); Phys. Rev. B 34, 1784 (1986); 38, 1613 (1988).

[6] G. Y. Guo and H. H. Wang, Chin. J. Phys. 38, 949 (2000).

[7] B. Heinrich et al., J. Vac. Sci. Technol. A 4, 1376 (1986); J. Cryst. Growth 81, 562 (1987).

[8] Z. Q. Wang, Y. S. Li, F. Jona, and P. M. Marcus, Solid State Commun. 61, 623 (1987).

[9] B. Heinrich et al., Phys. Rev. B 38, 12879 (1988).

[10] J.A.C. Bland et al., J. Magn. Magn. Mater. 93, 331 (1991).

[11] Z. Celinski et al., J. Magn. Magn. Mater. 166, 6 (1997).

[12] N. B. Brookes, A. Clarke, and P. D. Johnson, Phys. Rev. B 46, 237 (1992).

[13] J. I. Lee, S. C. Hong, A. J. Freeman, and C. L. Fu, Phys. Rev. B 47, 810 (1993).

[14] T. Lin et al., Phys. Rev. B 59, 13911 (1999).

[15] See, e.g., A. Aspelmeier et al., J. Magn. Magn. Mater. 146, 256 (1995); J. Wu and X. F. Jin, Phys. Rev. B 70, 212406 (2004).

[16] O. Rader et al., Phys. Rev. Lett. 72, 2247 (1994).

[17] W. X. Tang et al., J. Magn. Magn. Mater. 240, 404 (2002).

[18] Y.Z. Wu et al., J. Magn. Magn. Mater. 198-199, 297 (1999).

[19] See, for example, J.S. Claydon et al., Phys. Rev. Lett. 93, 037206 (2004).

[20] M. Tischer et al., Surf. Sci. 307-309, 1096 (1994).

[21] B. Schulz et al., Surf. Sci. 307-309, 1102 (1994); R. Zhang et al., Phys. Rev. Lett. 86, 2665 (2001).

[22] R. Mattheis and G. Quednau, J. Magn. Magn. Mater. 205, 143 (1999).

[23] Z. Tian et al., Phys. Rev. B 70, 012301 (2004).

[24] See, for example, F. Bensch et al., J. Appl. Phys. 91, 8754 (2002).

[25] See Derek Craik, Magnetism (University of Nottingham, Nottingham, UK, 1995).

[26] O. Thomas et al., Phys. Rev. Lett. 90, 017205 (2003).

[27] J. Buenemann, Europhys. Lett. 61, 667 (2003).

[28] X.F. Jin et al. (to be published). 